\title{
A New Neutrosophic Approach of Wiener Filtering for MRI Denoising
}

\author{
J. Mohan ${ }^{1}$, V. Krishnaveni' ${ }^{2}$, Yanhui Guo ${ }^{3}$ \\ ${ }^{1}$ Department of Electronics and Communication Engineering, P A College of Engineering and Technology, Pollachi, \\ Tamilnadu 642002, India, e-mail: jaimohan12@gmail.com \\ ${ }^{2}$ Department of Electronics and Communication Engineering, PSG College of Technology, Coimbatore, Tamilnadu 641004, \\ India, e-mail: vk@ece.psgtech.ac.in \\ ${ }^{3}$ School of Science, Technology and Engineering Management, Saint Thomas University, Miami Gardens, Florida 33054, \\ USA, e-mail: yguo@stu.edu
}

In this paper, a new filtering method based on neutrosophic set (NS) approach of wiener filter is presented to remove Rician noise from magnetic resonance image. A neutrosophic set, a part of neutrosophy theory, studies the origin, nature and scope of neutralities, as well as their interactions with different ideational spectra. Now, we apply the neutrosophic set into image domain and define some concepts and operators for image denoising. The image is transformed into NS domain, described using three membership sets: True (T), Indeterminacy (I) and False (F). The entropy of the neutrosophic set is defined and employed to measure the indeterminacy. The $\omega$-wiener filtering operation is used on $T$ and $F$ to decrease the set indeterminacy and remove noise. The experiments have conducted on simulated Magnetic Resonance images (MRI) from Brainweb database and clinical MR images corrupted by Rician noise. The results show that the NS wiener filter produces better denoising results in terms of visual perception, qualitative and quantitative measures compared with other denoising methods, such as classical wiener filter, the anisotropic diffusion filter, the total variation minimization scheme and non local means filter.

Keywords: Denoising, Magnetic Resonance imaging, Neutrosophic Set, Rician distribution, wiener.

\section{INTRODUCTION}

$\mathrm{M}$ AGNETIC RESONANCE imaging is powerful diagnostic technique used in radiology to visualize detailed internal structures of the human body. MRI is primarily used to demonstrate pathological or other physiological alterations of living tissues and is a commonly used form of medical imaging [1]. Despite significant improvements in recent years, MR images often suffer from low signal to noise ratio (SNR) especially in cardiac and brain imaging. Noise in MRI follows Rician Distribution [2] and thus limits the visual inspection and also this is problematic for further tasks such as segmentation of important features; classification of images for computer aided diagnostics, three dimensional image reconstruction and image registration. Therefore, it is important to improve the SNR of the images used during quantitative analysis. This is usually done by using a denoising method, which is an important preprocessing step used to improve the image quality by reducing the noise component while preserving all the image features.

Numerous approaches of denoising MR images have been proposed in the literatures. Henkelman [3] was the first to estimate the magnitude MR image from a noisy image. McVeigh et al. [4] proposed the spatial filtering and temporal filtering for reducing Gaussian noise in MR images. These filters eliminate the high frequency noise at the expense of blurring fine details and sharp edges in the MR images. A large number of edge-preserving methods have been proposed to overcome the above-mentioned blurring effects. For example, anisotropic diffusion filtering of MRI denoising proposed by Gerig et al. [5] are able to remove noise using gradient information while respecting important image structures. Recently, Krissian and Aja-
Fernandez [6] proposed a new anisotropic diffusion filter based on a linear minimum mean squared error estimation and partial difference equations for Rician noise removal that has achieved state-of-the-art results. Samsonov and Johnson [7] proposed the noise adaptive non-linear diffusion method for filtering MR images with spatially varying noise levels. By using the receiver coil sensitivity profiles, the priori information regarding the image noise level spatial distribution is obtained and it is utilized for the local adjustment of the anisotropic diffusion filter.

A simple wavelet based noise reduction was proposed by Weaver et al. [8]. The main drawback of the method was small structures similar in size to noise where also eliminated. Wavelet transform based denoising for Rician noise removal in MRI was proposed by Nowak [9]. Pizurica et al. [10] proposed an alternative for denoising MR images with Rician noise using low complexity joint detection and estimation method. In the squared magnitude of the MR image, introduce an analytical model for the probability of signal presence, which is adapted to the global coefficients histogram and to a local indicator of spatial activity (locally averaged magnitude of the wavelet coefficients). Subtract the constant bias from the scaling coefficients, and subsequently compute the square root of the denoised squared- magnitude image.

Bao and Zang [11] proposed a MR image denoising method based on an adaptive multiscale products threshold which incorporates the merits of interscale dependencies into the thresholding technique for denoising. Two adjacent wavelet subbands are multiplied to amplify the significant features and dilute noise. Apply thresholding to the multiscale products instead of the wavelet coefficients to differentiate the edge structures from noise better. The 
distribution of the products analyzed and an adaptive threshold is formulated to remove most of the noise. Thus, it is a good edge preserving denoising method. Yang and Fei [12] proposed the wavelet multiscale denoising method for the Rician nature of MR data. In this method, Radon transform is applied to the original MR images and the Gaussian noise model is used to process the MR sinogram image. A translation invariant wavelet transform is employed to decompose the MR sinogram into multiscales in order to effectively denoise the image. Rabbani [13] proposed the spatially adaptive wavelet based method which uses the maximum a posteriori (MAP) criterion on a local Laplacian prior for denoising low SNR MR images in order to enhance the visual quality.

Non local means (NLM) filter introduced by Buades et al. [14] has emerged as a very simple and an effective way to reduce noise while minimally affecting the original structures of the image. Manjon et al. [15] modified the original NLM algorithm to denoise the multispectral MRI. In the multispectral sequences, the similarity measure can be obtained by combining information of various channels. Manjon et al. [16] proposed the unbiased NLM approach for MRI denoising. The unbiased NLM is obtained by subtracting the noise bias from the squared value of NLM. The main drawback of the NLM algorithm is computational burden due to its complexity especially on 3D MRI data. In order to overcome this, Coupe et al. [17] proposed an optimized blockwise NLM filter for denoising 3D MRI. This is achieved by the following steps: an automatic tuning of the smoothing parameter, a selection of the most relevant voxels for NL means computation, a blockwise implementation and a parallelized computation.

Manjon et al. [18] proposed the adaptive NLM filter for denoising MR images with spatially varying noise levels, such as those obtained by parallel imaging and surface coil acquisitions. The information regarding the local image noise level is used to adjust the amount of denoising strength of the filter. Such information is automatically obtained from the images by using the local noise estimation method. This method does not require the prior knowledge of the coil sensitivity profiles in the MRI scanner. Manjon et al. [19] proposed the denoising methods for three dimensional MR images by exploiting the sparseness and self-similarity properties of the images. These methods are based on a three dimensional moving window cosine transform hard thresholding and a three dimensional rotationally invariant version of the NLM filter.

Lopez-Rubio and Florentine-Nunez [20] proposed a three dimensional (3D) MRI denoising method based on zeroth order 3D Kernel regression. In this method, the weighted average of the pixels are computed over a regression window and the weights are obtained from the similarities among small sized feature vectors associated to each pixel by using second order kernel regression. This local directional information about the weights guides the zeroth order 3D Kernel regressor to reconstruct the denoised MR image.

This paper focuses on developing a new denoising method based on the neutrosophic set (NS) approach of wiener filtering to remove the Rician noise in MRI. First the noisy
MRI is transformed into the neutrosophic set and the $\omega$ wiener filtering is employed to reduce the indetermination degree of the image, which is measured by the entropy of the indeterminate subset, after filtering, the noise will be removed.

\section{PROPOSED METHOD}

Neutrosophy, a branch of philosophy introduced in [21] as a generalization of dialectics, studies the origin, nature and scope of neutralities, as well as their interactions with different ideational spectra. In neutrosophy theory, every event has not only a certain degree of the truth, but also a falsity degree and an indeterminacy degree that have to be considered independently from each other [21]. Thus, a theory, event, concept, or entity, $\{A\}$ is considered with its opposite $\{A n t i-A\}$ and the neutrality $\{$ Neut $-A\}$. $\{$ Neut $-A\}$ is neither $\{A\}$ nor $\{A n t i-A\}$. The $\{$ Neut $-A\}$ and $\{$ Anti $-A\}$ are referred to as $\{$ Non $-A\}$. According to this theory, every idea $\{A\}$ is neutralized and balanced by $\{A n t i-A\}$ and $\{$ Non $-A\}$ [21]. NS provides a powerful tool to deal with indeterminacy.

Neutrosophic set had been applied to image thresholding, image segmentation and image denoising applications [2226]. Cheng and Guo [22] proposed a thresholding algorithm based on neutrosophy, which could select the thresholds automatically and effectively. The NS approach of image segmentation for real images [23] and for color texture image segmentation discussed in [24]. In [25], some concepts and operators were defined based on NS and applied for image denoising. It can process not only noisy images with different levels of noise, but also images with different kinds of noise well. The method proposed in [25] adapted and applied for MRI denoising [26], which shows some significant improvement in denoising. This paper is presented as an extended work of [27]; MRI denoising based on NS based wiener filtering is evaluated with clinical and sequence of MR images.

\subsection{Neutrophic Set.}

Let $U$ be a Universe of discourse and a neutrosophic set $A$ is included in $U$ An element $x$ in set $A$ is noted as $x(T, I, F) . \quad T, I$ and $F$ are called the neutrosophic components. The element $x(T, I, F)$ belongs to $A$ in the following way. It is $t \%$ true in the set, $i \%$ indeterminate in the set, and $f \%$ false in the set, where $t$ varies in $T, i$ varies in $I$ and $f$ varies in $F$.

\subsection{The image in neutrosophic set.}

Let $U$ be a Universe of discourse and $W$ is a set of $U$, which is composed by bright pixels. A neutrosophic image $P_{N S}$ is characterized by three membership sets $T, I, F$. A pixel $P$ in the image is described as $P(T, I, F)$ and belongs to $W$ in the following way: It is $t$ true in the set, $i$ indeterminate in the set, and $f$ false in the set, where 
$t$ varies in $T, i$ varies in $I$ and $f$ varies in $F$. Then the pixel $P(i, j)$ in the image domain is transformed into the neutrosophic set domain

$$
P_{N S}(i, j)=\{T(i, j), I(i, j), F(i, j)\} . T(i, j), I(i, j)
$$

and $F(i, j)$ are the probabilities belong to white pixels set, indeterminate set and non white pixels set respectively [2527], which are defined as:

$$
\begin{gathered}
T(i, j)=\frac{\bar{g}(i, j)-\bar{g}_{\text {min }}}{\bar{g}_{\max }-\bar{g}_{\min }} \\
\bar{g}(i, j)=\frac{1}{w \times w} \sum_{m=i-w / 2}^{i+w / 2} \sum_{n=j-w / 2}^{j+w / 2} g(m, n) \\
I(i, j)=\frac{\delta(i, j)-\delta_{\min }}{\delta_{\max }-\delta_{\min }} \\
\delta(i, j)=a b s(g(i, j)-\bar{g}(i, j)) \\
F(i, j)=1-T(i, j)
\end{gathered}
$$

where $\bar{g}(i, j)$ is the local mean value of the pixels of the window. $\delta(i, j)$ is the absolute value of difference between intensity $g(i, j)$ and its local mean value $\bar{g}(i, j)$.

\subsection{Neutrosophic image entropy.}

For an image, the entropy is utilized to evaluate the distribution of the gray levels. If the entropy is the maximum, the intensities have equal probability. If the entropy is small, the intensity distribution is non-uniform. Neutrosophic entropy of an image is defined as the summation of the entropies of three subsets $T, I$ and $F$ [2527]:

$$
\begin{gathered}
E n_{N S}=E n_{T}+E n_{I}+E n_{F} \\
E n_{T}=-\sum_{i=\min \{T\}}^{\max \{T\}} p_{T}(i) \ln p_{T}(i) \\
E n_{I}=-\sum_{i=\min \{I\}}^{\max \{I\}} p_{I}(i) \ln p_{I}(i) \\
E n_{F}=-\sum_{i=\min \{F\}}^{\max \{F\}} p_{F}(i) \ln p_{F}(i)
\end{gathered}
$$

where $E n_{T}, E n_{I}$ and $E n_{F}$ are the entropies of sets $T, I$ and $F$ respectively. $\quad p_{T}(i), \quad p_{I}(i)$ and $\quad p_{F}(i)$ are the probabilities of elements in $T, I$ and $F$ respectively, whose values equal to $i$.

\section{4. $\omega$ - wiener filtering operation.}

The values of $I(i, j)$ is employed to measure the indeterminate degree of element $P_{N S}(i, j)$. To make the set $I$ correlated with $T$ and $F$, the changes in $T$ and $F$ influence the distribution of element in $I$ and vary the entropy of $I$. A $\omega$ - wiener filtering operation for $P_{N S}, \hat{P}_{N S}(\omega)$, is defined as [27]:

$$
\begin{aligned}
& \hat{P}_{N S}(\omega)=P(\hat{T}(\omega), \hat{I}(\omega), \hat{F}(\omega)) \\
& \hat{T}(\omega)=\left\{\begin{array}{cc}
T & I<\omega \\
\hat{T}_{\omega} & I \geq \omega
\end{array}\right. \\
& \hat{T}_{\omega}(i, j)=\underset{(m, n) \in S_{i, j}}{\text { wiener }}\{T(m, n)\} \\
& \hat{F}(\omega)=\left\{\begin{array}{cc}
F & I<\omega \\
\hat{F}_{\omega} & I \geq \omega
\end{array}\right. \\
& \hat{F}_{\omega}(i, j)=\underset{(m, n) \in S_{i, j}}{\text { wiener }}\{F(m, n)\} \\
& \hat{I}_{\omega}(i, j)=\frac{\delta_{\hat{T}}(i, j)-\delta_{\hat{T} \min }}{\delta_{\hat{T} \max }-\delta_{\hat{T} \min }} \\
& \delta_{\hat{T}}(i, j)=\operatorname{abs}(\hat{T}(i, j)-\overline{\hat{T}}(i, j)) \\
& \overline{\hat{T}}(i, j)=\frac{1}{w \times w} \sum_{m=i-w / 2}^{i+w / 2} \sum_{n=j-w / 2}^{j+w / 2} \hat{T}(m, n)
\end{aligned}
$$

where $\delta_{\hat{T}}(i, j)$ is the absolute value of difference between intensity $\hat{T}(i, j)$ and its local mean value $\overline{\hat{T}}(i, j)$ at $(i, j)$ after $\omega$ - wiener filtering operation.

After new concepts and operators are defined in the neutrosophic set for an image, a MRI denoising method based on neutrosophic set approach of wiener filtering is proposed and summarized as below:

1) Transform the image into NS domain;

2) Use $\omega$ - wiener filtering operation on the true subset $T$ to obtain $T_{\omega}$;

3) Compute the entropy of the indeterminate subset $\hat{I}_{\omega}, E n_{\hat{I}_{\omega}}(i)$;

4) $\operatorname{if} \frac{E n_{\hat{I}_{\omega}}(i+1)-E n_{\hat{I}_{\omega}}(i)}{E n_{\hat{I}_{\omega}}(i)}<\delta$, go to Step 5; Else $T=\hat{T}_{\omega}$, go to Step 2;

5) Transform subset $\hat{T}_{\omega}$ from the neutrosophic domain into the gray level domain. 


\section{MATERIALS}

The experiments were conducted on two MRI datasets. The first data set consists of simulated MR images obtained from the Brainweb database [28]. The second data set consists of clinical MRI collected from PSG Institute of Medical Sciences and Research (PSG IMS \& R), Coimbatore, Tamilnadu, India. Simulated MR images are used collectively as the reference to evaluate and compare the validity of the proposed technique. It evicts the data dependency enabling precise comparative studies. The data set consists of $\mathrm{T} 1$ weighted Axial, T2 weighted Axial and T1 weighted Axial with multiple sclerosis (MS) lesion volumes of $181 \times 217 \times 181$ voxels (voxels resolution is $1 \mathrm{~mm}^{3}$ ), which are corrupted with different level of Rician noise ( $1 \%$ to $15 \%$ of maximum intensity). Rician noise added to the MR image was generated by Gaussian noise to real and imaginary parts and then computing the magnitude of the image.

In the clinical data sets, the images are acquired using Siemens Magnetom Avanto 1.5T Scanner.

a. T2 weighted Sagittal MR image of normal brain with TR $=4460 \mathrm{~ms}, \mathrm{TE}=85 \mathrm{~ms}, 5 \mathrm{~mm}$ thickness and $512 \times 512$ resolution.

b. T1 weighted Axial MR image of tumor pathology with $\mathrm{TR}=550 \mathrm{~ms}, \mathrm{TE}=8.7 \mathrm{~ms}, 5 \mathrm{~mm}$ thickness and $499 \times 559$ resolution.

\section{VALIDATION STRATEGIES}

The performance of the denoising algorithm is measured by using the quality metrics such as peak-signal-to-noise ratio (PSNR), the Structural Similarity (SSIM) index [29]. The peak signal to noise ratio in decibel $(\mathrm{dB})$ is measured using the following formula:

$$
P S N R=-10 \log _{10}\left[\frac{\sum_{i=0}^{i=H-1} \sum_{j=0}^{j=W-1}\left(I(i, j)-I_{d}(i, j)\right)^{2}}{H \times W \times 255^{2}}\right]
$$

where $I(i, j)$ and $I_{d}(i, j)$ represent the intensities of pixels $(i, j)$ in the original image and denoised image respectively. The higher the PSNR, the better the denoising algorithm is. SSIM gives the measure of the structural similarity between the original and the denoised images and are in the range of 0 to 1 . The SSIM works as follows: Let $x$ and $y$ be two non negative images, where as one has perfect quality. Then, the SSIM can serve as a quantitative measure of the similarity of the second image. The system separates the task of similarity measurement into three comparisons: luminance, contrast and structure. It can be defined as

$$
\operatorname{SSIM}(x, y)=\frac{\left(2 \mu_{x} \mu_{y}+C_{1}\right)\left(2 \sigma_{x y}+C_{2}\right)}{\left(\mu_{x}^{2}+\mu_{y}^{2}+C_{1}\right)\left(\sigma_{x}^{2}+\sigma_{y}^{2}+C_{2}\right)}
$$

where $\mu_{x}$ and $\mu_{y}$ are the estimated mean intensity and $\sigma_{x}$ and $\sigma_{y}$ are the standard deviations respectively. $\sigma_{x y}$ can be estimated as

$$
\sigma_{x y}=\frac{1}{N-1} \sum_{i=1}^{N}\left(x_{i}-\mu_{x}\right)\left(y_{i}-\mu_{y}\right)
$$

$C_{1}$ and $C_{2}$ in equation (19) are constants and the values are given as $C_{1}=\left(K_{1} L\right)^{2}$ and $C_{2}=\left(K_{2} L\right)^{2}$ where $K_{1}, K_{2}<<1$ is a small constant and $L$ is the dynamic range of the pixel values (255 for 8 bit gray scale images).

\section{RESULTS AND DISCUSSION}

\subsection{Performance Comparison.}

The performance of the proposed method has been compared with several standard and established algorithms.

\subsubsection{Wiener filter.}

$\mathrm{N}$. Wiener proposed the concept of wiener filtering also called as minimum mean square error filter or least square error filter in 1942 [30]. This method is founded on considering images and noise as random processes and the objective is to estimate $\hat{f}$ of the uncorrupted image $f$ such that the mean square error between them is minimized. This error measure is given by

$$
e^{2}=E\left\{(f-\hat{f})^{2}\right\}
$$

where $E\{\cdot\}$ is the expected value of the argument. In the frequency domain,

$$
\hat{F}(u, v)=\left[\frac{H^{*}(u, v) S_{f}(u, v)}{S_{f}(u, v)|H(u, v)|^{2}+S_{\eta}(u, v)}\right] G(u, v)
$$

where $H(u, v)$ is the transform degraded function, $H^{*}(u, v)$ is the complex conjugate of $H(u, v), S_{\eta}(u, v)$ is the power spectrum of the noise, $S_{f}(u, v)$ is the power spectrum of the undegraded image and $G(u, v)$ is the transform of the degraded image. The restored image in the spatial domain is given by the inverse transform of the frequency domain estimate $\hat{F}(u, v)$.

\subsubsection{Anisotropic Diffusion filter (ADF).}

Perona and Malik [31] introduced this method. In this approach the image $u$ is only convolved in the direction orthogonal to the gradient of the image which ensures the preservation of edges. The iterative denoising process of initial image $u_{0}$ can be expressed as 


$$
\left\{\begin{array}{l}
\frac{\partial u(x, t)}{\partial t}=\operatorname{div}(c(x, t) \nabla u(x, t)) \\
u(x, 0)=u_{0}(x)
\end{array}\right.
$$

where $\nabla u(x, t)$ is the image gradient at voxel $x$ and iteration $t, \quad \partial u(x, t) / \partial t$ is the partial temporal derivation of $u(x, t)$ and

$$
c(x, t)=g(\|\nabla u(x, t)\|)=e^{-\frac{\|\nabla u(x, t)\|}{K^{2}}}
$$

where $\mathrm{K}$ is the diffusivity parameter.

\subsubsection{Total variation (TV) minimization scheme.}

The difficult task to preserve edges while correctly denoising constant areas has been addressed also by Rudin, Osher and Fatemi. They proposed to minimize the TV norm subject to noise constraints [32], that is

$$
\hat{u}=\arg \min _{u \in \Omega^{3}} \int|\nabla u(x, t)| d x
$$

subject to

$$
\int_{\Omega^{3}}\left(u(x)-u_{0}(x)\right) d x=0 \text { and } \int_{\Omega^{3}}\left|u(x)-u_{0}(x)\right|^{2} d x=\sigma^{2}
$$

where $u_{0}$ is the original noisy image, $u$ is the restored image and $\sigma$ is the standard deviation of the noise. In this model, the TV minimization tends to smooth inside the image structures while keeping the integrity of boundaries. The TV minimization scheme can be expressed as an unconstrained problem

$$
\hat{u}=\arg \min _{u \in \Omega^{3}}\left[\int_{\Omega^{3}}|\nabla u(x)| d x+\lambda \int_{\Omega^{3}}\left|u(x)-u_{0}(x)\right|^{2} d x\right]
$$

where $\lambda$ is a Lagrange multiplier which controls the balance between the TV norm and the fidelity term. Thus $\lambda$ acts as the filtering parameter. Indeed, for high values for $\lambda$ the fidelity term is encouraged. For small values for $\lambda$ the regularity term is desired.

\subsubsection{Nonlocal means (NLM) filter.}

In the nonlocal means [14], Given a discrete noisy image $u=\{u(i) \mid i \in I\}$, the estimated value $N L[u](i)$, for a pixel $i$, is computed as a weighted average of all the pixels in the image,

$$
N L[u](i)=\sum_{j \in I} w(i, j) u(j)
$$

where the family of weights $\{w(i, j)\}_{j}$ depend on the similarity between the pixels $i$ and $j$, and satisfy the usual conditions $0 \leq w(i, j) \leq 1$ and $\sum_{j} w(i, j)=1$.

The similarity between two pixels $i$ and $j$ depends on the similarity of the intensity gray level vectors $u\left(N_{i}\right)$ and $u\left(N_{j}\right)$, where $N_{k}$ denotes a square neighborhood of fixed size and centered at a pixel $k$. This similarity is measured as a decreasing function of the weighted Euclidean distance, $\left\|u\left(N_{i}\right)-u\left(N_{j}\right)\right\|_{2, a}^{2}$, where $a>0$ is the standard deviation of the Gaussian kernel. The application of the Euclidean distance to the noisy neighborhoods raises the following equality

$$
E\left\|u\left(N_{i}\right)-u\left(N_{j}\right)\right\|_{2, a}^{2}=\left\|u\left(N_{i}\right)-u\left(N_{j}\right)\right\|_{2, a}^{2}+2 a^{2}
$$

This equality shows the robustness of the algorithm since in expectation the Euclidean distance conserves the order of similarity between pixels.

The pixels with a similar grey level neighborhood to $u\left(N_{i}\right)$ have larger weights in the average. These weights are defined as,

$$
w(i, j)=\frac{1}{Z(i)} e^{-\frac{\left\|u\left(N_{i}\right)-u\left(N_{j}\right)\right\|_{2, a}^{2}}{h^{2}}}
$$

where $Z(i)$ is the normalizing constant

$$
Z(i)=\sum_{j} e^{-\frac{\left\|u\left(N_{i}\right)-u\left(N_{j}\right)\right\|_{2, a}^{2}}{h^{2}}}
$$

and the parameter $h$ acts as a degree of filtering. It controls the decay of the exponential function and therefore the decay of the weights as a function of the Euclidean distances.

All the methods were implemented using MATLAB 2010a (The Math Works, Inc) in Windows XP 64-bit Edition (Pentium Dual core $2.4 \mathrm{GHz}$ with 4GB of RAM). Wiener filtering was computed using the function from Matlab Image Processing Toolbox [33]. For ADF, the parameter K varies from 0.05 to 1 with a step of 0.05 and the number of iterations varies from 1 to 15 . For TV minimization, the parameter $\lambda$ varies from 0.01 to 1 with step of 0.01 and the number of iterations varies from 1 to 10 . There are three key 
parameters that need to be set to use the NLM filter; one is the size of the search window $(w)$, second one is the size of the neighborhood window $(f)$ and finally, the degree of the filtering $(h)$. For the simulation, the parameters have chosen as $w=5, f=2$ and $h$ is proportional to the noise level of the image [14].

\subsection{Evaluation on simulated data set.}

The detailed images of the denoising results obtained for the T1 weighted and T2 weighted MR images corrupted by $7 \%$ and $9 \%$ of Rician noise respectively shown in Fig.1. and Fig.2. These figures are provided the visual comparison of the denoising results for different filters. From these results, the proposed method surpassed all other methods at high noise levels on the three types of data in terms of producing more detailed denoised image in which all the distinct features and small structural details are well preserved.

The PSNR and the SSIM values obtained for the T1 weighted and T2 weighted MR images with different noise levels (1\% to $15 \%$ ) using the aforementioned denoising techniques are given in Fig.3. As the level of noise increase, the performance of the proposed NS wiener filter shows significant improvement over the other denoising methods. Table 1. shows a comparison of the experimental results for the denoising methods based on PSNR and SSIM for the T2 weighted, T1 weighted with MS lesion and T1 weighted axial images corrupted by $7 \%, 9 \%$ and $15 \%$ respectively. Higher the value of PSNR and higher the value of SSIM shows that the proposed filter perform superior than the other denoising methods.

\subsection{Evaluation on clinical data set.}

The detailed images of the denoising results obtained for the T2 weighted Sagittal MR image are given in Fig.4. The detailed features and edges in the image are well preserved by the proposed method compared with other denoising methods. The denoising results obtained for the small part of T1 weighted axial brain with tumor pathology are shown in Fig.5. With the other denoising methods (ADF, Wiener, TV and NLM), the sharpness along the edges and the pathology structure is extensively blurred. Comparing the NLM and NS wiener, it is evident that NS wiener preserves well the details. This confirms that NS wiener is superior with respect to PSNR and SSIM.

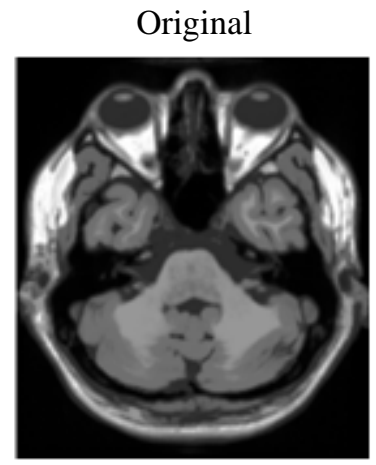

TV

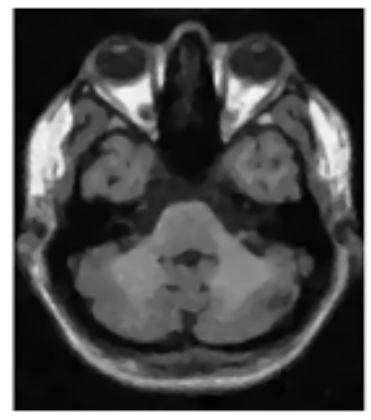

Noisy

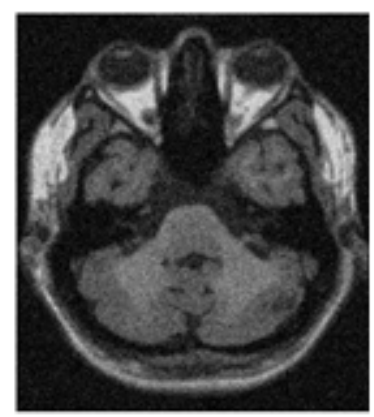

NLM

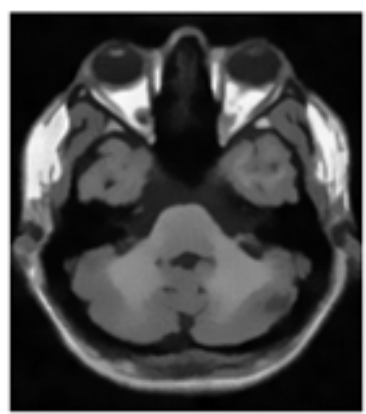

ADF
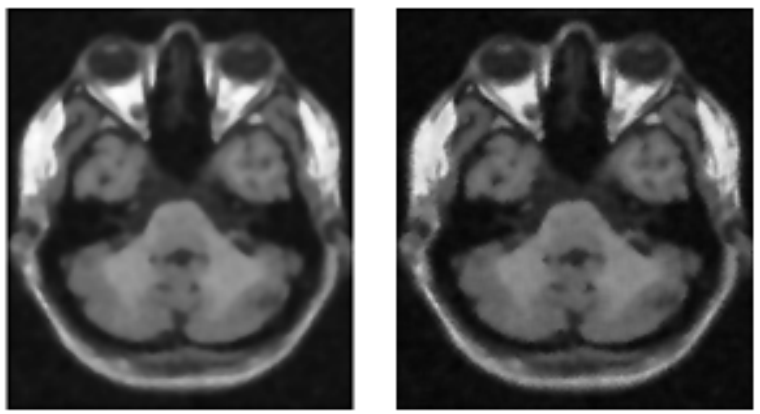

NS Wiener

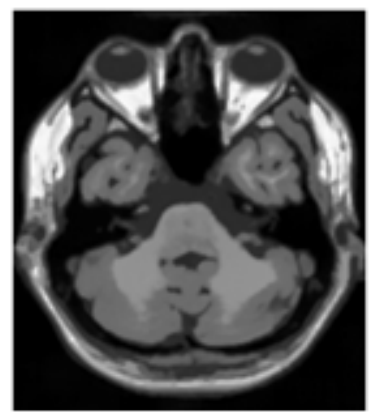

Fig.1. Denoising results of simulated T1 weighted axial MRI corrupted by $7 \%$ of Rician noise. 


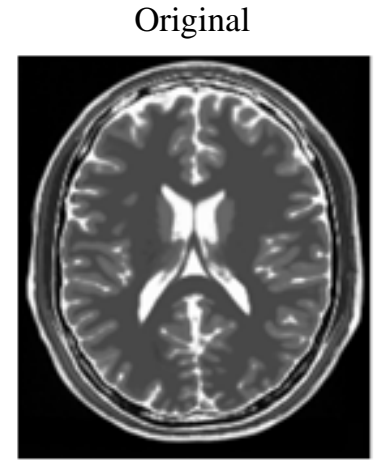

TV

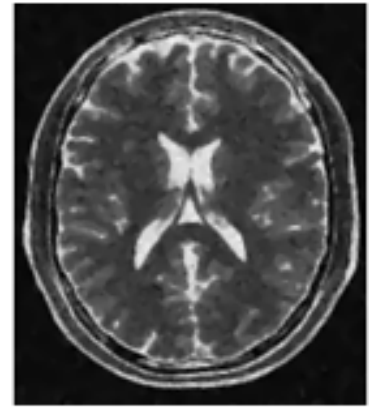

Noisy

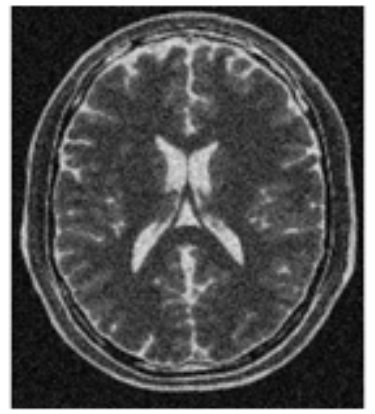

NLM

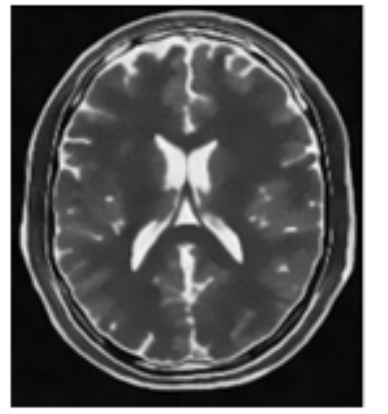

ADF

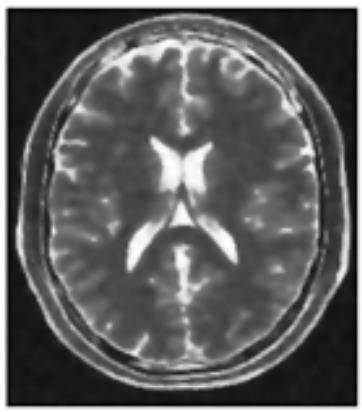

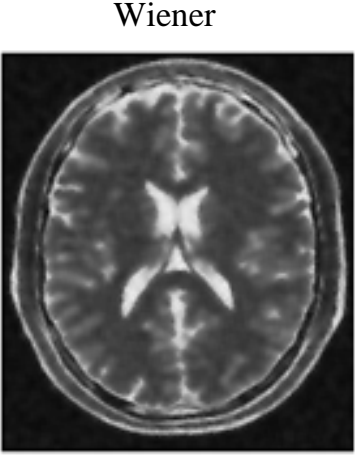

NS Wiener

Fig.2. Denoising results of simulated T2 weighted axial MRI corrupted by 9\% of Rician noise.
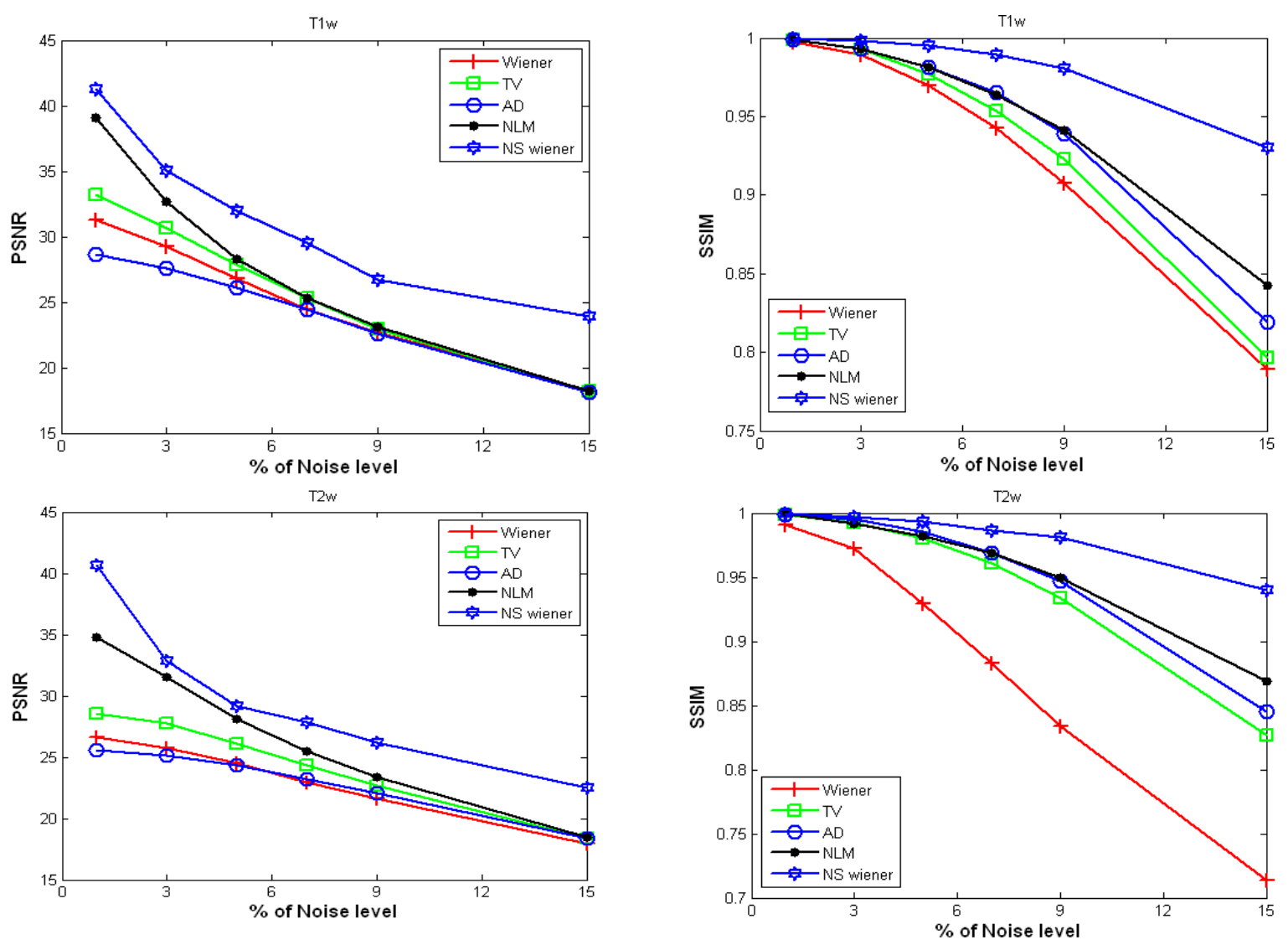

Fig.3. Comparison results for Brainweb simulated MR images. Left: PSNR of the compared methods for different image types and noise levels. Right: SSIM of the compared methods for different image types and noise levels. 


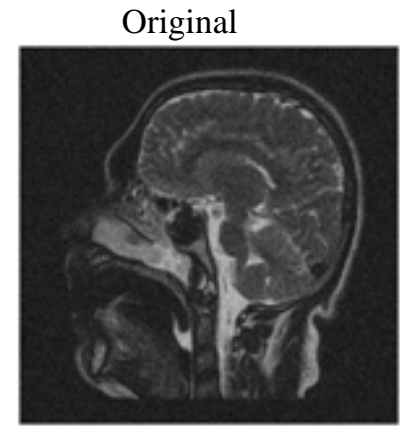

TV

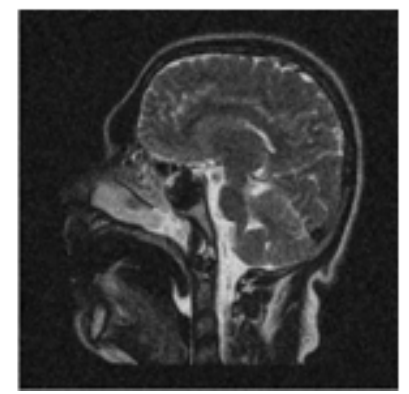

ADF

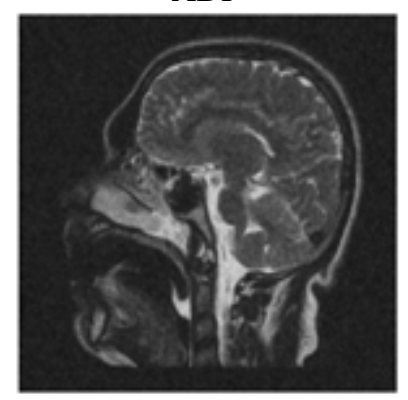

NLM

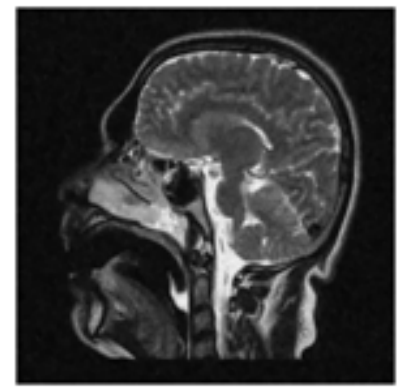

Wiener

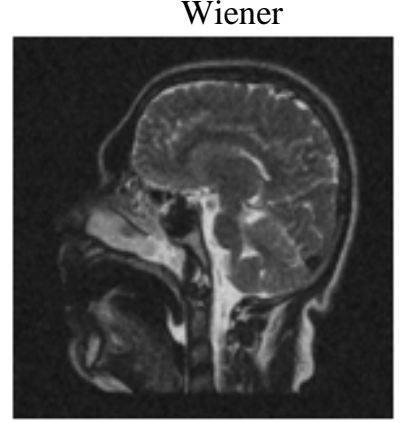

NS Wiener

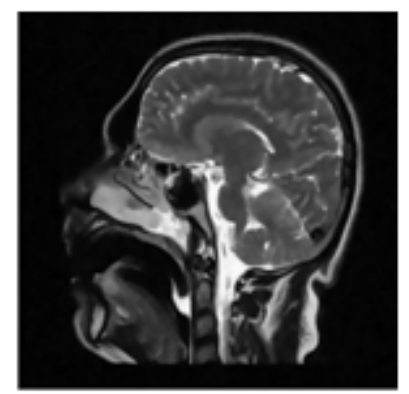

Fig.4. Denoising results of clinical T2 weighted Sagittal MRI.

Original

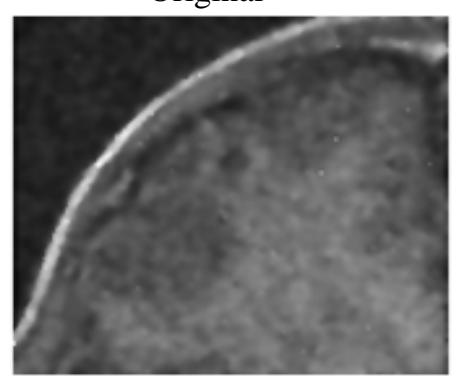

TV

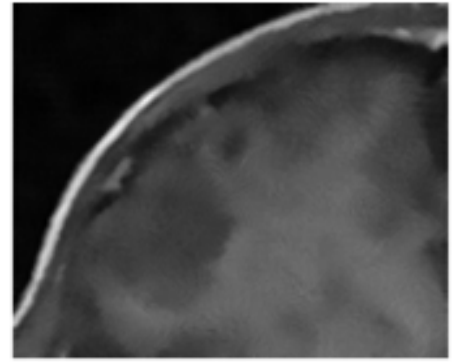

ADF

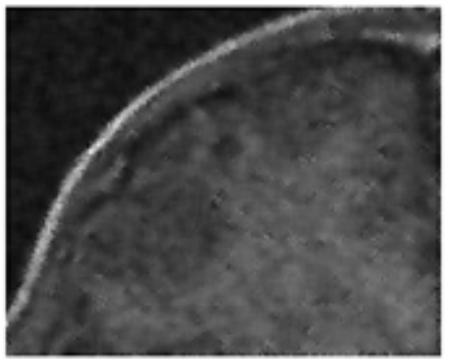

NLM

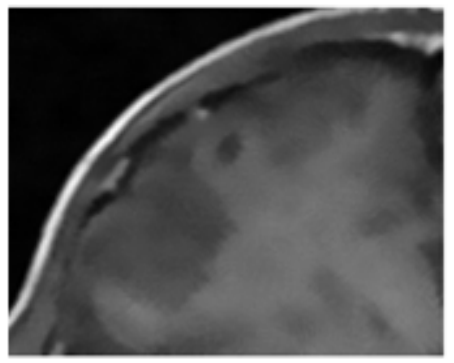

Wiener

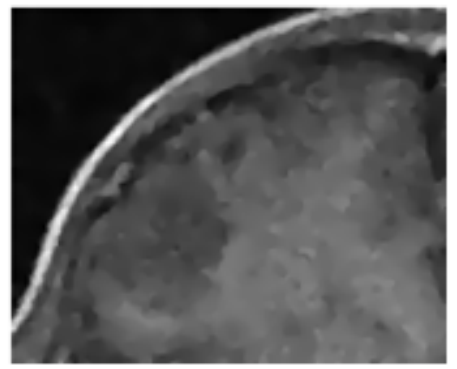

NS Wiener

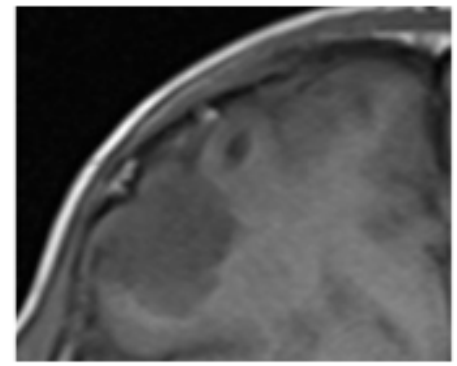

Fig.5. Denoising results of small part of the clinical T1 weighted axial MRI with tumor pathology. 
Table 1. Comparison of the denoising techniques based on the performance metrics for simulated MR images.

\begin{tabular}{|c|c|c|c|}
\hline \multirow{2}{*}{ MR Image } & \multirow{2}{*}{$\begin{array}{l}\text { Denoising } \\
\text { Methods }\end{array}$} & \multicolumn{2}{|c|}{ Performance Metrics } \\
\hline & & $\operatorname{PSNR}(\mathrm{dB})$ & SSIM \\
\hline \multirow{5}{*}{$\begin{array}{l}\text { T2- Weighted } \\
\text { brain MRI } \\
\text { corrupted by 7\% } \\
\text { Rician noise }\end{array}$} & Wiener & 22.98 & 0.8832 \\
\hline & TV & 24.37 & 0.9606 \\
\hline & $\mathrm{ADF}$ & 23.21 & 0.9686 \\
\hline & NLM & 25.52 & 0.9687 \\
\hline & NS Wiener & 27.89 & 0.9865 \\
\hline \multirow{5}{*}{$\begin{array}{l}\text { T1- Weighted } \\
\text { brain MRI with } \\
\text { MS lesion } \\
\text { corrupted by 9\% } \\
\text { Rician noise }\end{array}$} & Wiener & 21.07 & 0.9068 \\
\hline & TV & 21.43 & 0.9208 \\
\hline & $\mathrm{ADF}$ & 21.05 & 0.9368 \\
\hline & NLM & 21.44 & 0.9389 \\
\hline & NS Wiener & 27.68 & 0.9802 \\
\hline \multirow{5}{*}{$\begin{array}{l}\text { T1- Weighted } \\
\text { brain MRI } \\
\text { corrupted by } \\
\text { 15\% Rician } \\
\text { noise }\end{array}$} & Wiener & 18.19 & 0.7892 \\
\hline & TV & 18.20 & 0.7966 \\
\hline & $\mathrm{ADF}$ & 18.15 & 0.8191 \\
\hline & NLM & 18.22 & 0.8426 \\
\hline & NS Wiener & 23.86 & 0.9305 \\
\hline
\end{tabular}

\section{CONCLUSION}

In this paper, a novel MRI denoising technique based on neutrosophic set approach of wiener filtering has been proposed. The method is adapted to Rician noise characteristics. The image is described as a NS set using three membership sets $T, I$ and $F$. The entropy in neutrosophic image domain is defined and employed to measure the indetermination. The wiener filter is applied to reduce the set's indetermination and to remove the noise in the MR image.

The performance of the proposed denoising filter is compared with wiener, ADF, TV and NLM based on PSNR and SSIM. The experimental results demonstrate that the proposed approach can remove noise automatically and effectively. This filtering method tends to produce good denoised image not only in terms of visual perception but also in terms of the quality metrics. In the clinical MRI with tumor pathology, the filter preserves the major visual signature of the given pathology. And finally, further works should be implementing spatially adaptive wiener filtering in the neutrosophic domain for accounting the spatially varying noise level in parallel imaging MRI.

\section{ACKNOWLEDGMENT}

The authors would like to thank Dr. V. Maheswaran of PSG Institute of Medical Sciences and Research (PSG IMS\&R) Coimbatore, Tamilnadu, India for providing us the clinical MRI data and for providing his opinion on the diagnostic details of the denoised images.

\section{REFERENCES}

[1] Wright, G. (1997). Magnetic resonance imaging. IEEE Signal Process Magazine, 14 (1), 56-66.

[2] Gudbjartsson, H., Patz, S. (1995). The Rician distribution of noisy MRI data. Magetic Resonance in Medcine, 34 (6), 910-914.

[3] Henkelman, R.M. (1985). Measurement of signal intensities in the presence of noise in MR images. Medical Physics, 12 (2), 232-233.

[4] McVeigh, E.R., Henkelman, R.M., Bronskill, M.J. (1985). Noise and filtration in magnetic resonance imaging. Medical Physics, 12 (5), 586-591.

[5] Gerig, G., Kubler, O., Kikinis, R., Jolesz, F.A. (1992). Nonlinear anisotropic filtering of MRI data. IEEE Transaction on Medical Imaging, 11 (2), 221-232.

[6] Krissian, K., Aja-Fernández, S. (2009). Noise driven anisotropic diffusion filtering of MRI. IEEE Transaction on Image Processing, 18 (10), 2265-2274.

[7] Samsonov, A., Johnson, C. (2004). Noise-adaptive nonlinear diffusion filtering of MR images with spatially varying noise levels. Magnetic Resonance in Medicine, 52 (4), 798-806.

[8] Weaver, J.B., Xu, Y., Healy, D.M., Cromwell, L.D. (1991). Filtering noise from images with wavelet transforms. Magnetic Resonance Imaging, 21 (2), 288-295.

[9] Nowak, R.D. (1999). Wavelet-based Rician noise removal for magnetic resonance imaging. IEEE Transaction on Image Processing, 8 (10), 1408-1419.

[10] Pizurica, A., Philips, W., Lemahieu, I., Acheroy, M. (2003). A versatile wavelet domain noise filtration technique for medical imaging. IEEE Transaction on Medical Imaging, 22 (3), 323-331.

[11] Bao, P., Zhang, L. (2003). Noise reduction for magnetic resonance images via adaptive multiscale products thresholding. IEEE Transaction on Medical Imaging, 22 (9), 1089-1099.

[12] Yang, X., Fei, B. (2011). A wavelet multiscale denoisng algorithm for magentic resonance images, Measurement Science and Technology, 22 (2), 1-12.

[13] Rabbani, H., (2011). Statistical modeling of low SNR magnetic resonance images in wavelet domain using Laplacian prior and two-sided Rayleigh noise for visual quality improvement. Measurement Science Review, 11 (4), 125-130.

[14] Buades, A., Coll, B., Morel, J.M. (2005). A review of image denoising algorithms with a new one. Multiscale Modeling and Simulation, 4 (2), 490-530.

[15] Manjon, J.V., Robles, M., Thacker, N.A. (2007). Multispectral MRI de-noising using non-local means. In Medical Image Understanding and Analysis 2007 (MIUA), 17-18 July 2007. University of Wales Aberystwyth, 41-46.

[16] Manjón, J.V., Carbonell-Caballero, J., Lull, J.J., García-Martí, G., Martí-Bonmatí, L., Robles, M. (2008). MRI denoising using non-local means. Medical Image Analysis, 12 (4), 514-523. 
[17] Coupe, P., Yger, P., Prima, S., Hellier, P., Kervrann, C., Barillot, C. (2008). An optimized blockwise nonlocal means denoising filter for 3-D magnetic resonance images. IEEE Transaction on Medical Imaging, 27 (4), 425-441.

[18] Manjón, J.V., Coupe, P., Martí-Bonmatí, L., Collins D.L., Robles, M., (2010). Adaptive non-local means denoising of MR images with spatially varying noise levels. Magnetic Resonance Imaging, 31 (1), 192-203.

[19] Manjón, J.V., Coupe, P., Buades, A., Collins, D.L., Robles, M. (2012). New methods for MRI denoising based on sparseness and self-similarity. Medical Image Analysis, 16 (1), 18-27.

[20] Lopez-Rubio, E., Florentin-Nunez, M.N. (2011). Kernel regression based feature extraction for 3D MR image denoising. Medical Image Analysis, 15 (4), 498513.

[21] Samarandache, F. (2003). A Unifying Field in Logics: Neutrosophic Logic. Neutrosophy, Neutrosophic Set, Neutrosophic Probability (third edition). American Research Press.

[22] Cheng, H.D., Guo, Y. (2008). A new neutrosophic approach to image thresholding. New Mathematics and Natural Computation, 4 (3), 291-308.

[23] Guo, Y., Cheng, H.D. (2009). New neutrosophic approach to image segmentation. Pattern Recognition, 42 (5), 587-595.

[24] Sengur, A., Guo, Y. (2011). Color texture image segmentation based on neutrosophic set and wavelet transformation. Computer Vision and Image Understanding, 115 (8), 1134-1144.

[25] Guo, Y., Cheng, H.D., Zhang, Y. (2009). A new neutrosophic approach to image denoising. New Mathemetics and Natural Computation, 5 (3), 653662.
[26] Mohan, J., Krishnaveni, V., Guo, Y. (2011). A neutrosophic approach of MRI denoising. In International Conference on Image Information Pocessing (ICIIP), 3-5 November 2011. IEEE, 1-6.

[27] Mohan, J., Krishnaveni, V., Guo, Y., Kanchana, J. (2012). MRI denoising based on neutrosophic wiener filtering. In IEEE International Conference on Imaging Systems and Techniques (IST), 16-17 July 2012. IEEE, 327-331.

[28] Kwan, R.K., Evans, A.C., Pike, G.B. (1999). MRI simulation based evaluation of image processing and classification methods. IEEE Transaction on Medical Imaging, 18 (11), 1085-1097.

[29] Wang, Z., Bovik, A.C., Sheikh, H.R., Simoncell, E.P. (2004). Image quality assessment: From error visibility to structural similarity. IEEE Transaction on Image Processing, 13 (4), 600-612.

[30] Gonzalez, R.C., Woods, R.E. (2002). Digital Image Processing (second edition). Prentice Hall.

[31] Perona, P., Malik, J. (1990). Scale-space and edge detection using anisotropic diffusion. IEEE Transaction on Pattern Analysis and Machine Inteligence, 12 (7), 629-639.

[32] Rudin, L.I., Osher, S., Fatemi, E. (1992). Nonlinear total variation based noise removal algorithms. Physica D: Nonlinear Phenomena, 60 (1-4), 259-268.

[33] Mathworks. The Matlab image processing toolbox. http://www.mathworks.com/access/helpdesk/help/tool box/images/ 